\title{
Independence and Ignorance: How Agnotology Informs Set-Theoretic Pluralism
}

\author{
Neil Barton ${ }^{1}$
}

Received: 8 February 2017/ Accepted: 23 February 2017/Published online: 11 March 2017

(C) The Author(s) 2017. This article is published with open access at Springerlink.com

\begin{abstract}
Introduction Much of the discussion of set-theoretic independence, and whether or not we could legitimately expand our foundational theory, concerns how we could possibly come to know the truth value of independent sentences. This paper pursues a slightly different tack, examining how we are ignorant of issues surrounding their truth. We argue that a study of how we are ignorant reveals a need for an understanding of settheoretic explanation and motivates a pluralism concerning the adoption of foundational theory.

Materials and Methods Our strategy is as follows. First ("Varieties of Independence"), we note two different kinds of independence, those that are sensitive to large cardinal axioms, and those that are not. We pick two well-studied examples from the literature: \{Cantor's Continuum Hypothesis\} (henceforth $\mathrm{CH}$ and \{Projective Determinacy\} (henceforth 'PD'). We then ("Multiversism and Pluralism") present two views concerning set-theoretic ontology (namely Multiversism and Universism), and explain how each might be linked to the acceptance or rejection of a Pluralism in set-theoretic foundations. Next ("Varieties of Ignorance"), we change tack and exposit some literature on the study of ignorance (often called agnotology). We then ("What is our Ignorance of Independent Sentences Like?"), examine how various positions might regard our ignorance of $\mathrm{CH}$ and PD. Finally ("How Ignorance Affects Pluralism"), we argue that certain views of our ignorance of independent sentences motivate pluralism concerning the study of set-theoretic foundations, even on a Universist picture.
\end{abstract}

The author wishes to thank Michèle Friend, Paul Hoyningen-Huene, Colin Rittberg, and an anonymous referee for helpful discussion in preparing the paper. He is also very grateful for the generous support of the FWF (Austrian Science Fund) through Project P 28420.

Neil Barton

neil.barton@univie.ac.at

1 Kurt Gödel Research Center for Mathematical Logic (KGRC), Währinger Straße, 25, 1090 Vienna, Austria 
Conclusion We conclude that despite the prima facie tension between Universism and Pluralism, the character of our ignorance suggests a fusion of the two positions.

Keywords Philosophy of mathematics - Philosophy of set theory · Multiversism · Foundations of mathematics · Agnotology

\section{Introduction}

It is well known that many statements of set theory cannot be either proved or disproved on the basis of the ZFC axioms. Some have seen this as indicative of a failure of our concept of set to determine a single Universe of sets rather than a Multiverse of different universes (we shall see some discussion of these views later).

Much of the discussion surrounding independence focusses on whether or not we could come to know set-theoretic sentences independent of $\mathbf{Z F C}$, and if so, how. ${ }^{1}$ In this paper, we examine a slightly different question: what is our ignorance of independent sentences like? Assuming that we do not know the answers to questions independent from $\mathbf{Z F C}$, how should we understand this ignorance? How, if at all, might this influence any pluralism concerning set-theoretic foundation?

Our strategy is as follows. First ("Varieties of Independence") we note two different kinds of independence, those that are sensitive to large cardinal axioms, and those that are not. We pick two well-studied examples from the literature: Cantor's Continuum Hypothesis (henceforth ' $\mathrm{CH}$ ') and Projective Determinacy (henceforth 'PD'). We then ("Multiversism and Universes") present two views concerning set-theoretic ontology, and explain how each might be linked to the acceptance or rejection of a Pluralism in set-theoretic foundations. Next ("Varieties of Ignorance"), we change tack and exposit some literature on the study of ignorance (often called agnotology). We then ("What is Our Ignorance of Independent Sentences Like?") examine how various positions might regard our ignorance of $\mathrm{CH}$ and PD. Finally ("How Ignorance Affects Pluralism"), we argue that certain views of our ignorance of independent sentences motivates pluralism concerning the study of set-theoretic foundations, even on a Universist picture. We conclude that despite the prima facie tension between Universism and Pluralism, the character of our ignorance suggests a fusion of the two positions.

\section{Varieties of Independence}

Before we go further we should be precise about two different kinds of independence we might see. A natural question, once one is aware of Cantor's Theorem that there is no bijection between a set and its powerset, is whether or not there is an intermediate cardinality between that of the natural numbers and its powerset. The hypothesis that there is no such cardinal number is known as Cantor's

\footnotetext{
${ }^{1}$ See, for example, the work of Maddy in Maddy (1988a, b, 2011), and Koellner in Koellner (2010). For a pessimistic yet elegant position, see Hamkins (2012) and Hamkins (2015).
} 
Continuum Hypothesis (or ' $\mathrm{CH}$ ') and can be coded as a statement of third-order arithmetic.

However, independence from ZFC comes before the level of third-order arithmetic, yielding a different kind of independence. Determinacy axioms concern strategies (represented as functions) for generating reals, and can be played over certain subsets of the reals (where one player wins if the real generated is in the relevant subset, where the other wins if the real is not in the subset). For example, Projective Determinacy is the statement that any projectively defined set of reals has a winning strategy. PD can be coded as a (schematic) statement of second-order arithmetic. $^{2}$

There are a number of arguments both for and against $\mathrm{CH}$ and PD in themselves. ${ }^{3}$ Important for our purposes, however, will be the relationship between these principles and large cardinals. ${ }^{4}$ There is no formal definition of a large cardinal axiom; however, there are a family of natural principles that index consistency strength, seemingly in a linear fashion. ${ }^{5}$ Salient is the following theorem:

\section{Theorem 1 Martin and Steel (1989) If there are infinitely many Woodin cardinals ${ }^{6}$ then PD holds.}

\footnotetext{
${ }^{2}$ For details of Projective Determinacy, as well as other Determinacy Axioms, see Jech (2002) and Koellner and Woodin (2010). For the reader interested in the technical details: The kind of game in question involves two players (denoted by ' $I$ ' and ' $I I$ ') in a state of perfect information (i.e. both $I$ and $I I$ have infallible knowledge about past moves of the game). The game is played over some subset $S$ of ${ }^{\omega} \omega$ : the set of all infinite sequences of natural numbers. For the purposes of determinacy, ${ }^{\omega} \omega$ is used to represent the set of all real numbers; since it is homeomorphic to the irrationals, one can simply prove results about $\omega^{\omega}$ and then transfer the theorems across using the homeomorphism. $I$ and $I I$ takes turns to play natural numbers against one another. After $\omega$-many turns in this game, the players will have generated a real number (let it be denoted by ' $r$ '). $I$ is said to win if $r \in S$, and player $I I$ wins if $r \notin S$. We can see that the possible moves allowed in a game are represented by a tree $T$, given by the legitimate moves players may make at each successive point of the game, with a length ascribed to each position of a play of the game $p$ (denoted by 'length $\left.(p)^{\prime}\right)$. A strategy for player $A$ is a function $\sigma$ with domain $\{p \in T \mid[$ length $(\mathrm{p})$ is even and $\mathrm{p}$ is not a terminal element of $\mathrm{T}]\}$, such that $\sigma(p)$ is always a legal move for $A$ in $T$. Similarly we can define the notion of a strategy for $B$ as a function $\tau$ with domain $\{p \in T \mid[$ length $(\mathrm{p})$ is odd and $\mathrm{p}$ is not a terminal element of $\mathrm{T}]\}$ such that $\tau(p)$ is always a legal move for $A$ in $T$. A winning strategy for player $X$ is a strategy $\pi$ for $X$ such that $X$ wins every game consistent with $\pi$. A set of reals is said to be determined iff one of the two players has a winning strategy. The projective sets are sets of reals obtained from the operations of complementation and projection from closed subsets of $\left({ }^{\omega} \omega\right)^{n}$ for $n \in \omega$. Thus, PD states that whenever the subset $S$ over which the game is being played is projective, then one of the two players has a winning strategy.

${ }^{3}$ An excellent review of some of the options is discussed in Maddy (1988a, b).

${ }^{4}$ Of course, large cardinal axioms themselves constitute a significant and interesting area of independence from ZFC. While we provide some remarks later concerning large cardinals and mathematical explanation, we are largely interested here in independence low in the cumulative hierarchy.

${ }^{5}$ While all evidence points in this direction, nonetheless, due to the informal nature of the notion of large cardinal axiom, no a priori proof of this claim is possible.

${ }^{6}$ A cardinal $\kappa$ is Woodin iff for all $A \subseteq V_{\kappa}$, there are arbitrarily large $\alpha<\kappa$ such that for all $\beta<\kappa$ there exists an elementary embedding $j: V \longrightarrow \mathfrak{M}$ with critical point $\alpha$, such that $j(\alpha)>\beta, V_{\beta} \subseteq M$, and $A \cap V_{\beta}=A \cap j\left(V_{\beta}\right)$.
} 
Thus we see that PD is sensitive to the existence of large cardinals. ${ }^{7}$ By contrast, we have the following theorem concerning $\mathrm{CH}$ :

Theorem 2 Lévy and Solovay (1967) Let $\mathfrak{M}$ be a model of set theory and $\kappa$ be measurable. ${ }^{8}$ Then there are forcing extensions of $\mathfrak{M}$ in which $\mathrm{CH}$ is true and others in which $\mathrm{CH}$ is false but $\kappa$ remains measurable.

The above theorem shows that known large cardinal hypotheses cannot be used to settle the truth value of $\mathrm{CH}$ in the manner of PD. This is because, no matter how many large cardinals we have, we are able to use a relatively mild small forcing to modify the truth value of $\mathrm{CH}$, while leaving the large cardinal properties intact. As we shall see, this difference will prove to be relevant. Many scholars feel that this difference between PD and $\mathrm{CH}$ means that we are in a very different epistemic position with respect to each. ${ }^{9}$

\section{Multiversism and Pluralism}

Independence has motivated several theories concerning how we should understand the subject matter of set theory. Central to our discussion will be the following two positions:

Multiversism is the view that there are many universes of set theory, no one of which is ontologically privileged. Any universe of sets can be extended to a larger universe.

Universism is the view that there is a single, unique, maximal universe of settheoretic discourse.

often Multiversism is seen as linked to the following position.

Pluralism is the view that we should investigate many different set theories, and no one is foundationally privileged in the sense that we conduct foundational inquiry in different theories at different times.

Multiversism and Pluralism appear to be naturally linked. If we are Multiversists and believe that there are a variety of universes, each of which satisfies some theory or other and is on an ontologically equal footing, then we are likely to hold that the theory of sets we adopt is underdetermined. We can operate within any particular legitimate universe, using the theory that one finds there as our foundation. For example, Hamkins writes:

The multiverse view does not abandon the goal of using set theory as an epistemological and ontological foundation for mathematics, for we expect to

\footnotetext{
7 In fact this sensitivity is shared by several other statements of second-order arithmetic at the level of $V_{\omega+1}$ (for example $A D^{L(\mathbb{R})}$ ), in contrast to third-order arithmetic (which inhabits $V_{\omega+2}$ ). See Koellner and Woodin (2010) for details.

8 A cardinal $\kappa$ is measurable iff it is the critical point of a non-trivial elementary embedding $j: V \longrightarrow \mathfrak{M}$. It is the weakest kind of cardinal defined by an elementary embedding from $V$ to an inner model thereof.

9 See, for example, Maddy (2011) and Koellner (2006).
} 
find all our familiar mathematical objects, such as the integer ring, the real field and our favourite topological spaces, inside any one of the universes of the multiverse. On the multiverse view, set theory remains a foundation for the classical mathematical enterprise. The difference is that when a mathematical issue is revealed to have a set-theoretic dependence, as in the independence results, then the multiverse response is a careful explanation that the mathematical fact of the matter depends on which concept of set is used, and this is almost always a very interesting situation, in which one may weigh the desirability of various set-theoretic hypotheses with their mathematical consequences. (Hamkins 2012, p. 419)

Thus, Hamkins has a position where we can operate in any one of a number of different universes of sets. Despite the fact that many of these universes satisfy different theories, we can nonetheless use them as foundational as all the relevant objects studied by the 'ordinary' mathematician appear there.

While someone who believes in the existence of a single, unique, maximal universe of sets might well be interested in a diversity of different theories, they nonetheless hold that there is a fact of the matter concerning which one is true. Thus, they might think that they should be interested in cutting down the theories available (eventually focussing on one such) in order to get closer to a better account of the truth value of independent sentences. As we shall see, a study of how we are ignorant of sentences independent from $\mathbf{Z F C}$ reveals that this methodology is misguided. In order to understand the Universe of sets better, and strengthen the case for one's own favourite theory of sets, it is fruitful pursue a wide variety of other foundational theories.

\section{Varieties of Ignorance}

Let us take stock. We have seen that several authors regard our epistemic limitations concerning sets as indicative of the existence of a multiverse of sets rather than a single universe thereof. This link merits examination. Most philosophers have focussed on what it would take to come to know (or, more minimally, be justified in asserting that) $\mathrm{CH}$. For example, Maddy writes:

The question of how the unproven can be justified is especially pressing in current set theory, where the search is on for new axioms to determine the size of the continuum. This pressing problem is also the deepest that contemporary mathematics presents to the contemporary philosopher of mathematics. Not only would progress towards understanding the process of mathematical hypothesis formation and confirmation contribute to our philosophical understanding of the nature of mathematics, it might even be of help and solace to those mathematicians actively engaged in the axiom search. (Maddy 1988a, p. 482)

arguing that we should analyse the process of confirmation and justification. Her ideas are developed further in Maddy (1990, 1997, 2007, 2011), and similar ideas 
have also been taken up by Koellner and Woodin in Koellner (2006) and Koellner and Woodin (2010). In the opposite direction, Hamkins argues that any attempt to come to know or justify $\mathrm{CH}$ is doomed to fail:

I claim that our extensive experience in the set-theoretic worlds in which $\mathrm{CH}$ is true and others in which $\mathrm{CH}$ is false prevents us from looking upon any statement settling $\mathrm{CH}$ as being a natural set-theoretic truth. We simply have had too much experience by now with the contrary situation. (Hamkins 2015, p. 135)

Hamkins' point is thus that the use of various model-theoretic constructions in proving independence results facilitates an ability to understand what it is like to reason in those worlds. This then prevents any widespread acceptance of $\mathrm{CH}$; as soon as an axiom is shown to imply $\mathrm{CH}$ or its negation, its naturalness is immediately vitiated by excluding prima facie natural set-theoretic universes.

While most philosophers have focussed on the back and forth of this debate, and what it would take to come to know or justify independent sentences, a positive account of our ignorance appears lacking. The issue is especially relevant given the advances in the philosophical study of ignorance that have been made over the last 30 years. Before we embark on a more detailed analysis of the agnotological status of independent sentences, we first provide a brief exposition of the relevant literature necessary for our arguments. ${ }^{10}$

The first kind of ignorance we shall consider is that of conscious ignorance. Such ignorance concerns questions ${ }^{11}$ to which we do not know the answers, but we nonetheless know that we do not know. Such questions are typically the targets of our investigations (both scientific and otherwise). Good examples (from my own extensive ignorance database) include:

(1.) I do not know how many miles my dad's car has on the clock.

(2.) I do not know what parts of category theory were used in the first proofs of Fermat's Last Theorem.

(3.) As of the year 2010, I did not know whether or not there were Higgs bosons.

(4.) I do not know whether or not there are infinitely many twin primes.

Each case has a number of differences. (1.) I do not know, simply because I do not find the question especially interesting. Despite this, I could easily verify it (say by checking his odometer next time I get a lift). (2.) I do not know, however, presumably with enough time and effort I could learn the required mathematics, and there definitely are people who do know. (3.) and (4.) are both no fault of mine, but I do know what would constitute/would have constituted a solution in a particular direction in each case (namely an observation of the relevant particle at CERN for the Higgs boson, and a peer-reviewed proof for the Twin Prime Conjecture).

\footnotetext{
${ }^{10}$ An excellent introduction and survey is available in Wilholt.

11 Ignorance is often formulated as directed towards research questions rather than propositions in order to avoid thorny issues surrounding Meno's paradox. See Wilholt (2017) for discussion.
} 
Despite these vagaries in kinds of ignorance, however, I do know (1) that I do not know the answer to a particular question, and (2) roughly what it would take to have an answer. Thus my ignorance is conscious: I know about it, and can investigate accordingly. ${ }^{12}$ More precisely, we can follow Bromberger (1992) in providing necessary conditions on ignorance of an agent $A$ with respect to some research question $Q$ :

(A) $A$ is able to articulate $Q$ in a language in which she is competent and is aware of $Q$.

(B) $A$ does not know the answer to $Q$.

(C) $Q$ admits of a single correct answer.

(D) $A$ believes that she does not know the correct answer to $Q$.

(E) $A$ believes that $Q$ has a correct answer.

Conscious ignorance can come in different flavours, however. Consider, for example, the following question:

\section{(5.) What happens when an object goes into a black hole?}

I am consciously ignorant of this question. My lack of ignorance is especially interesting, however, in that it plausibly displays the following two features ${ }^{13}$ :

$A$ is in a $p$-predicament with regard to $Q$ if and only if, in $A$ 's views, $Q$ admits of a right answer, but $A$ can think of no answer to which, in $A$ 's views, there are no decisive objections.

and

$A$ is in a $b$-predicament with regard to $Q$ if and only if the question is sound, but the correct answer is beyond what the person can conceive or articulate.

Why am I in a $b$-predicament and a $p$-predicament with respect to (5.)? Well, for those explanations I understand concerning what happens when we observe something enter a black hole, I can think of good reasons to reject each. Every explanation of which I am aware, I find deeply problematic on the basis of some gedanken experiment or other. Thus I am in a $p$-predicament. However, I am also likely to be in a $b$-predicament; whatever the correct explanation is, it is likely that, with my limited knowledge of esoteric theoretical physics, I am currently unable to compose or understand the answer, even if someone directly tells me.

$p$-predicaments and $b$-predicaments are independent phenomena; any combination of them is possible. For example, though I might be in a $b$-predicament and $p$ -

\footnotetext{
12 The examples of (3.) and (4.) are somewhat subtle in that for practical reasons I have to rely on the expertise of others to provide verification. However, should I be inclined to, I could (in some appropriate modal sense) try and investigate the issue, and know what would constitute a solution in each case. For example, I could give up philosophy tomorrow and begin to attempt to prove the Twin Prime Conjecture.

13 See Bromberger (1992), Chap. 2 for a fuller description of these kinds of phenomena, as well as Wilholt (2017).
} 
predicament with respect to the question of black holes, I am not in either position with respect to the number of miles on the odometer of my dad's car. I can think of many plausible values, for which I do not have a robust reason to think false. Moreover, I will be able to articulate what the value will be: some relatively small natural number. Returning again to the question of black holes, though I am probably in both a $b$-predicament and a $p$-predicament, things could quite easily have been otherwise. Suppose I think that the view that the surface of a black hole acts as a hologram of its contents is at least plausible, ${ }^{14}$ and am not aware of the various gedanken experiments that challenge such a view. Thus, I am not in a $p$-predicament: I can think of no decisive objections against the view that the surface of a black hole behaves like a hologram of its contents. However, I may very well still be in a $b$ predicament (say if the actual answer turns out to be very complex). For the converse direction, suppose that the holographic account of what happens when an object falls into a black hole is actually correct. Then I would be in a p-predicament (I still regard the holographic explanation as deeply problematic), but not a $b$-predicament, I can (just about) cognise and formulate what happens when an object falls into a black hole. When we are either in a $p$-predicament or $b$-predicament and consciously ignorant, we will say that we are deeply consciously ignorant. ${ }^{15}$

In addition to conscious ignorance, we also have opaque ignorance. This concerns questions to which we do not know the answer, and we also do not know that we do not know the answer. In each of the cases we will violate one of conditions (A), (C), (D), or (E). We might be ignorant of the answer to question because we are unable to articulate the question properly (and hence would find ourselves automatically in a $b$-predicament as well). It might be that we fail to recognise that the question does not admit of a correct answer. Alternatively, we might just be in a state of error, believing that we have an answer to the question when actually our answer is incorrect. Finally, we might regard $Q$ as lacking a correct answer, when it actually possesses one.

It should be noted that opacity does not necessarily imply depth. For example, suppose that my dad's car has 30,000 miles on the clock. Suppose further that I snuck a quick glance at the odometer yesterday, however I misread the ' 3 ' as a ' 2 ', and hence I believe that his car has done only 20,000 miles. What should we say about this case? I am opaquely ignorant: I believe (wrongly) that there are 20,000 miles on the clock, violating (D). However, I am in neither a $p$-predicament nor a $b$ predicament: I think there is an answer to which there are no good objections (namely 20,000 miles), and I can perfectly well articulate the correct answer. ${ }^{16}$

\footnotetext{
${ }^{14}$ See Susskind (1994) for an exposition of this fun idea.

15 Wilholt (2017) reserves the use of the term 'deep' solely for cases when we find ourselves in a $p$ predicament. As we shall see, $b$-predicaments will also be relevant here, so I will opt for a more liberal usage.

16 Examples of this sort bear a resemblance to Gettier-style cases, but for the fact that the belief in question is false. For example, if we modified the above example so that I still misread the ' 3 ' as a ' 2 ', but in fact someone has wound the clock forward by 10,000 miles (so my dad's car actually has done 20,000 miles), we would arrive at a standard Gettier case: my belief is true, I have justification, but I cannot reasonably be said to know. As we shall see, forms of mathematical Gettier-style situations (and the variety of ignorance they engender) will be relevant for our arguments.
} 


\section{What is Our Ignorance of Independent Sentences Like?}

The time has come to put the recent developments explained above to work in coming to a better understanding of our ignorance of independent sentences. A Multiversist attacks the problem by using their view of ontology to dispel much of our ignorance. Our knowledge concerning sentences such as $\mathrm{CH}$ consists in how they behave across the Multiverse. Such a sentiment receives expression in the work of Multiversists:

On the multiverse view, consequently, the continuum hypothesis is a settled question; it is incorrect to describe the $\mathrm{CH}$ as an open problem. The answer to $\mathrm{CH}$ consists of the expansive, detailed knowledge set theorists have gained about the extent to which it holds and fails in the multiverse, about how to achieve it or its negation in combination with other diverse set-theoretic properties. Of course, there are and will always remain questions about whether one can achieve $\mathrm{CH}$ or its negation with this or that hypothesis, but the point is that the most important and essential facts about $\mathrm{CH}$ are deeply understood, and these facts constitute the answer to the $\mathrm{CH}$ question. (Hamkins 2012, p. 429)

Thus, for a liberal multiversist of Hamkins' persuasion, while there is some ignorance regarding how $\mathrm{CH}$ behaves in certain models, largely speaking we have a good deal of knowledge regarding $\mathrm{CH}$.

The cases that we shall examine concern attitudes to $\mathrm{CH}$ and PD given the Universist's picture of set-theoretic ontology. ${ }^{17}$ The first task is to settle on the research question with which we are interested. The most immediate questions would be:

(6.) What is the value of the continuum?

(7.) Does every projective set of reals admit of a winning strategy?

However, we can never be in either a $p$-predicament or $b$-predicament with respect to (6.) or (7). For (6.), we can articulate any value the continuum might take (and so cannot be in a $b$-predicament), and also it is one of the main lessons of the independence phenomenon that there are many values the continuum might take that cannot be (currently) reasonably objected to (and so we are not in a $p$ predicament). For (7.) we note that as a simple true or false question, we can easily articulate the correct answer even if we do not know it (I just did), and again it is not the case that both responses admit of strong seemingly defeating objections. Thus, our ignorance regarding either $\mathrm{CH}$ or PD themselves is shallow, and in itself cannot be used in coming to understand $\mathrm{CH}$ or $\mathrm{PD}$ better. A reformulation of the requisite research question is required in order to bring agnotology to bear. Fortunately, the

\footnotetext{
17 There is a subtle question of how things go on multiversist pictures that are not as liberal as Hamkins' (see, for example, Steel 2014 and Arrigoni and Friedman 2013). Though an expansion of the project should accommodate these cases, for the sake of argumentative clarity we choose to focus on the Universist position here.
} 
above two questions are not the only research questions involved in the project of justifying new axioms for set theory. A famous quotation from Gödel is helpful here:

For if the meanings of the primitive terms of set theory...are accepted as sound, it follows that the set-theoretical concepts and theorems describe some well-determined reality, in which Cantor's conjecture must be either true or false. Hence its undecidability from the axioms being assumed today can only mean that these axioms do not contain a complete description of that reality. Such a belief is by no means chimerical, since it is possible to point out ways in which the decision of a question, which is undecidable from the usual axioms, might nevertheless be obtained. (Gödel 1964, p. 260)

Important to Gödel's argument, and indeed subsequent discussions of the justification of new axioms of set theory, is that we desire some way of achieving a more complete description of the Universe. How can this be done? Discussing certain axioms, Gödel writes:

These axioms show clearly, not only that the axiomatic system of set theory as used today is incomplete, but also that it can be supplemented without arbitrariness by new axioms which only unfold the content of the concept of set explained above. ( Gödel 1964, pp. 260-261)

justifications then, for Gödel, should respond to some feature of the universe of sets. It is this feature of the Universe of sets, that explains ${ }^{18}$ why the continuum has the value it does, or why every projective set of reals admits of a winning strategy. This focus on explanation indeed is partly in the target of Bromberger (1992)'s analysis of $p$-predicaments and $b$-predicaments. Thus we may phrase our question (schematically) as follows:

(8.) Let $\phi$ be some set-theoretic sentence independent from ZFC. What feature (suitably axiomatised) of the universe of sets explains which of $\phi$ and $\neg \phi$ is true?

Now, we can be in either a $p$-predicament or $b$-predicament with respect to this question. Let us consider each of CH and PD in turn. Of course, one's ignorance of (or indeed lack thereof) $\mathrm{CH}$ and PD is dependent upon the attitudes and epistemic features of the agent in question. A salient problem here is that we can only examine ignorance with respect to an individual set theorist, and finding consensus across the community is difficult. We will, therefore, examine the different ways that ignorance may play out, and what should be said in each case.

A very important difference between the two principles is that PD is at least claimed to be known (or at least believed with a high degree of credence) by several set theorists and philosophers. The fact that large cardinals imply determinacy axioms, and that determinacy axioms imply the existence of inner models with large

\footnotetext{
18 It is, of course, an exceptionally tricky issue how to provide a full account of mathematical explanation. We shall set this issue aside here.
} 
cardinals has been seen by several authors ${ }^{19}$ as good evidence for the truth of PD. So, as an answer to (8.), many set theorists believe that:

(8.A) The network of results between determinacy axioms and Woodin cardinals is good evidence for the truth of certain large cardinal axioms, and the fact that the universe supports these cardinals explains why PD is true.

It is a difficult question whether or not such set theorists would claim to know the truth value of PD. In what follows, to make our arguments clear, we will simply assume this. $^{20}$ The situation with $\mathrm{CH}$ is markedly different. Though there are various projects aiming at finding a resolution of the continuum problem, few set theorists would claim that they know the answer.

Suppose then that $\phi$ is one of $\mathrm{CH}$ or PD, and that the agent does not believe that she knows the answer to what explains whether or not $\phi$. Might we be in a $p$ predicament with respect to the explanation of $\phi$ ?

The answer will depend on the extent to which one views the extant resolutions of $\phi$ as admitting defeating counter arguments. I think, however, it is fair to say that each of the competing resolutions of $\mathrm{CH}$ or PD might be true. It would seem like a highly pessimistic agent to say that each admits of defeating objections in her eyes. Thus, while it is possible that we are in a $p$-predicament, it is possibly the less interesting of the two phenomena.

We may very well be in a $b$-predicament with respect to (8.), however. It is entirely plausible that the resolution to an independent sentence requires substantial additional conceptual machinery, one that it might not be possible for us to articulate given our current epistemic situation. Thus, especially with respect to $\mathrm{CH}$, the possibility of a $b$-predicament should both be acknowledged and examined.

Suppose on the other hand that the agent believes that she does know the answer with respect to (8.) and either $\mathrm{CH}$ or PD holds (though, as noted above, it is more likely that PD is the target here). In that case, she may be correct, and (8.) might indeed be her preferred explanation for the truth of the relevant $\phi$. However, in this situation, one can still envisage that the person might be wrong that they know the sentence, say if the sentence holds but the universe does not conform to their desired explanation. Here, the agent is in a variety of Gettier-style situation with respect to PD (or $\mathrm{CH}$ ). The relevant sentence is true but their justification is defective (in that their explanation makes false claims about the universe). It is thus in their beliefs about their justifications and explanations where the ignorance lies. ${ }^{21}$ In such a situation, they would be opaquely ignorant. Moreover, they may well find themselves in a $b$-predicament: the answer to the research question might be

\footnotetext{
19 See Koellner and Woodin (2010) for a survey of this literature.

${ }^{20}$ We could have, instead, just moved to the nearest possible world at which the agent does believe that she knows in order to examine the case there.

21 The example is interesting in its own right, as it creates problems for accounts of knowledge that make use of possible worlds. For, many such accounts make use of a sensitivity constraint: in possible worlds in which the sentence is false, the agent does not believe it. However, on the widely held view that mathematical objects exist out of necessity if at all, it is difficult to make sense of this requirement. For, on the assumption that that a mathematical sentence $\phi$ is true (or false) there are no possible worlds in which $\phi$ is false (or true).
} 
beyond what the agent can conceive. Indeed, if we are generous to set theorists and philosophers of set theory, we might think that should a truly correct and compelling answer be discovered, it will ultimately be accepted.

Thus, we see that within the Universist's framework there are several options. For a given independent sentence $\phi$, and question of the form of (8.), we might be in one of the following three situations: either (1) we know the answer to (8.), (2) our ignorance is either conscious, or (3) we are opaquely ignorant in virtue of holding a misplaced confidence in our erroneous reasons for holding $\phi$ true. Assuming that we are in fact ignorant (so in cases (1) or (2)), there is then the separate question of whether or not we are in a $b$-predicament with respect to the independent sentence. Moreover, in both the case of (2) and (3), there are reasons to think that a $b$ predicament is at least possible, if not likely.

\section{How Ignorance Affects Pluralism}

Let us return to the case of PD. One might think that we should be, on a Universist's picture, in a less pernicious epistemic situation with PD compared to $\mathrm{CH}$. We have the Martin-Steel Theorem that PD follows from the existence of infinitely many Woodin cardinals, and also know that axioms of definable determinacy reverse to yield inner models of the large cardinals. We seem to have a wealth of information in the case of PD that is not possessed in the $\mathrm{CH}$ case.

Note, however, that we are more likely to be opaquely ignorant with respect to PD, given the Martin-Steel Theorem. If it turns out that there are not the requisite Woodin cardinals (let's say the existence of a Woodin cardinal turns out to be inconsistent), yet we are currently staunch adherents to the view that PD holds in virtue of the existence of infinitely many Woodin cardinals, then we would, as agents, be opaquely ignorant in virtue of erroneously holding ourselves to be knowers. This goes for other programmes or traditions too. It might simply turn out that the tradition in which the agent is steeped is simply not correct concerning $V$, yielding opaque ignorance (assuming, of course, that they have not rebelled against their tradition!). In fact, the mere existence of incompatible foundational set-theoretic programmes, implies (assuming that there are at least some who take themselves to know on each side) that there must be such opaque ignorance for some agents. In contrast, assuming that we are consciously ignorant of (8.) with respect to some sentence, we may very well hold that the correct explanation is not something we can currently articulate.

The key fact to note is that in each case, determining whether or not one is in a $b$ predicament is key, and if we are in a $b$-predicament investigation and resolution of our $b$-predicament aids in the search for new axioms. In the case of PD, part of shoring up our confidence in PD on the basis of infinitely many Woodin cardinals is determining that there is not some hitherto unrecognised aspect of the Universe of sets that vitiates our explanation. Similarly, one might think that with respect to why $\mathrm{CH}$ or $\neg \mathrm{CH}$ holds, we are likely to be in a $b$-predicament. The only way this $b$ predicament can be rectified is by developing and working within new foundational proposals. The importance of determining our $b$-predicaments with respect to set theory is thus important for engaging in the justificatory process. For, if we believe 
that we know why $\phi$ holds, then analysing alternative pictures in detail helps to rule them out and narrows the chance of there being a hitherto unrecognised aspect of the universe of sets, and if we think that we do not know whether or not $\phi$, it is entirely plausible that we are in a $b$-predicament and so should be open to new and revolutionary set-theoretic axioms. This sort of process is one to which agnotologists are sensitive:

Here are some examples of such questions that have at some point played a role in periods of massive theoretical and conceptual change: "What would we observe if we chased alongside a light beam at the speed of light?", "Under what conditions are two events that occur at different points in space simultaneous?", "What happens when two freely falling heavy bodies are connected in mid-fall?", "Why does the electron in an $H$ atom not spiral into the core, emitting radiation of greater and greater frequency?" These questions were, I submit, understandable even before the respective episodes of revolutionary change that they are associated with had occurred. It was in taking them seriously and pursuing them (amongst other questions) that Einstein, Galileo and Bohr encountered deep-seated problems which led them to attempt radical theoretical-conceptual adjustments. These adjustments, in turn, enabled them to pursue other, novel questions, thus opening up whole new areas of conscious ignorance that had been thoroughly opaque before (Wilholt 2017, pp. 16-17).

The point is that by analysing our $b$-predicaments and removing them, we can come to fruitful conceptual change, converting much opaque ignorance into conscious ignorance. Importantly however, this conversion is not just intrinsically interesting, but also helps inform our confidence in our currently held beliefs. For example, suppose that a new set theoretic principle $\Psi$ is proposed, one which both seems natural and to which we were previously in a $b$-predicament. Suppose this principle also has the property that it implies PD and reverses to inner models of large cardinals. This increases our confidence in our explanation of the truth of PD; areas of opaque ignorance towards which we were previously in a $b$-predicament turn out to mesh nicely with our overall picture. Suppose on the other hand that there are some phenomena we wish to explain and $\Psi$ seems both natural and implies these data. We come to hold the belief that $\Psi$ explains the phenomena and we were previously in a $b$-predicament with respect to this fact. However, suppose that it then turns out that $\Psi$ implies that there are no Woodin cardinals. Our initial picture of the explanation and justification of PD would be disconfirmed and might lead us to reform our epistemological and agnotological stance towards PD and its explanation.

What is the pluralistic upshot? Simply that much of philosophical discourse concerning set theory has been in promotion of one or other conception of the nature of the set-theoretic universe, to the possible detriment of other projects and foundational theory. Our analysis questions the extent to which this is a fruitful ${ }^{22}$ methodology.

\footnotetext{
22 The concept of fruitfulness has been the focus of a good deal of recent work in the philosophy of mathematics recently (such as in Tappenden 2008 and Maddy 2011). We do not mean anything too deep (or precise) by the term: simply that this methodology might not be the best or most effective.
} 
Certainly views have to be defended against objections, and often doing so is the best way of filling them out in full detail. If, however, this is pursued solely for the promotion of one set of axioms over others we run into two difficulties. First, as discussed above, even if one has a favourite position concerning the nature of the universe of sets, the study of alternative frameworks helps to reassure oneself that one is not in an erroneous opaque $b$-predicament with respect to the view. Secondly, the resolution of $b$-predicaments in a manner compatible with one's position results in truly novel observations concerning $V$, thereby helping one to better situate one's position. However, in coming to a resolution of a $b$-predicament, even one that ends up being resolved in a manner compatible with one's own view, we may require a period of toleration of other foundational systems in tension with one's current position. After all, it is precisely the nature of $b$-predicaments that we cannot yet formulate the relevant solution. Exploration of these issues thus requires novel techniques, some of which may conflict with our own view while being necessary to see the shape of a solution. Thus, somewhat paradoxically, one's own view can be strengthened (both through understanding our knowledge/ignorance and our level of confidence in our justifications/explanations) by adopting a Pluralism towards foundational theory in order to draw out the contents of different ways of looking at the universe. The development of alternative frameworks yields information about one's own preferred theory (indeed many other theories) and helps us to tell a better story of how different axiom systems stand with respect to confirmation. Fixation on a single theory masks this useful information and obscures possible unorthodox pioneering insights.

\section{Conclusion}

Let us take stock. We identified in "Varieties of Independence" two main kinds of independence from ZFC; those for which we believe we have a well-justified response (using large cardinals) and those which we find more perplexing. We then argued in "Multiversism and Pluralism" that often Multiversism and Pluralism are linked. In "Varieties of Ignorance" we reviewed some of the agnotological literature and noted the difference between conscious ignorance, opaque ignorance, articulated through the notions of $p$-predicaments and $b$-predicaments. In "What is Our Ignorance of Independent Sentences Like?" we provided an analysis of how we might be ignorant of explanations of the truth or falsity of sentences independent from ZFC. We argued that our ignorance may be either opaque or conscious, shallow or deep depending on the agent. It was also argued, however, that $b$ predicaments could have a special role to play in our understanding of independence; through examining our $b$-predicaments we both shore up held beliefs and attack our conscious ignorance effectively. This focus on the possibility of $b$-predicaments motivates a pluralism about foundational theory, ${ }^{23}$ even on a Universist understanding of the subject matter of set theory. While the Universist

\footnotetext{
23 There is a very deep question here, one we do not have the space to address, of how agnotology might inform Pluralism concerning the language in which we express our foundational theory. We might consider, for example, whether agnotology has any implications for the 'debate' between set-theoretic, category-theoretic, and homotopy-type-theoretic foundations.
} 
may think that one theory is true, the adoption of different foundational theories is an effective way of yielding epistemological information about each.

We close with a remark concerning directions for future research. While it has been argued that the Universist has some reason to accept Pluralism, it remains to be seen how independence relates to the study of ignorance on other ontological frameworks (e.g. the various species of Multiversism). Moreover, we selected the two very narrow cases of PD and $\mathrm{CH}$, a full examination of our ignorance of other kinds of independence (such as the independence of large cardinals) may also be in order. For the moment, however, it seems that the philosophical study of ignorance has interesting insights for the search for new axioms in set theory.

Acknowledgements Open access funding provided by University of Vienna.

Open Access This article is distributed under the terms of the Creative Commons Attribution 4.0 International License (http://creativecommons.org/licenses/by/4.0/), which permits unrestricted use, distribution, and reproduction in any medium, provided you give appropriate credit to the original author(s) and the source, provide a link to the Creative Commons license, and indicate if changes were made.

\section{References}

Arrigoni, T., \& Friedman, S.-D. (2013). The hyperuniverse program. Bulletin of Symbolic Logic, 19, 77-96.

Bromberger, S. (1992). On what we know we don't know: Explanation, theory, linguistics, and how questions shape them. Chicago: University of Chicago Press.

Gödel, K. (1964). What is Cantor's continuum problem? In [?] (pp. 254-270). : Oxford University Press. Hamkins, J. D. (2012). The set-theoretic multiverse. The Review of Symbolic Logic, 5(3), 416-449.

Hamkins, J. D. (2015). Is the dream solution of the continuum hypothesis attainable? Notre Dame Journal of Formal Logic, 56(1), 135-145.

Jech, T. (2002). Set theory. Heidelberg: Springer.

Koellner, P. (2006). On the question of absolute undecidability. Philosophia Mathematica, 14, 153-188.

Koellner, P. (2010). On the question of absolute undecidability. In S. Feferman, C. Parsons, \& S. G. Simpson (Eds.), Kurt Gödel: essays for his centennial (pp. 189-222). Poughkeepsie: Association for Symbolic Logic.

Koellner, P., \& Woodin, H. (2010). Large cardinals from determinacy. Handbook of set theory (pp. 1951-2119). Heidelberg: Springer.

Lévy, A., \& Solovay, R. (1967). Measurable cardinals and the continuum hypothesis. Israel Journal of Mathematics, 5(4), 234-248.

Maddy, P. (1988a). Believing the axioms I. The Journal of Symbolic Logic, 53(2), 481-511.

Maddy, P. (1988b). Believing the axioms II. The Journal of Symbolic Logic, 53(3), 736-764.

Maddy, P. (1990). Realism in mathematics. Oxford: Clarendon Press.

Maddy, P. (1997). Naturalism in mathematics. Oxford: Oxford University Press.

Maddy, P. (2007). Second philosophy. Oxford: Oxford University Press.

Maddy, P. (2011). Defending the axioms. Oxford: Oxford University Press.

Martin, D. A., \& Steel, J. R. (1989). A proof of projective determinacy. Journal of the American Mathematical Society, 2(1), 71-125.

Steel, J. (2014). Gödel's program. In J. Kennedy (Ed.), Interpreting Gödel. Cambridge: Cambridge University Press.

Susskind, L. (1994). The world as a hologram. arXiv:hep-th/9409089.

Tappenden, J. (2008). Mathematical concepts: Fruitfulness and naturalness. In P. Mancosu (Ed.), The philosophy of mathematical practice (pp. 276-301). Oxford: Oxford University Press.

Wilholt, T. (2017). On knowing what one does not know: Ignorance and the aims of research. In J. Kourany, \& M. Carrier (Eds.), Agnotology: Ways of producing, preserving, and dealing with ignorance (forthcoming). 